\title{
Improvement of molecular adhesion for spatial applications at Thales SESO over the past 20 years
}

Natacha Cocheteau, Christian Du Jeu, Alizee Loarer, Patrick Robert, Mailys Thorigny

Natacha Cocheteau, Christian Du Jeu, Alizee Loarer, Patrick Robert, Mailys Thorigny, "Improvement of molecular adhesion for spatial applications at Thales SESO over the past 20 years," Proc. SPIE 11852, International Conference on Space Optics - ICSO 2020, 118521N (11 June 2021); doi: $10.1117 / 12.2599287$

SPIE Event: International Conference on Space Optics - ICSO 2021, 2021, Online Only 


\section{International Conference on Space Optics-ICSO 2020}

Virtual Conference

30 March-2 April 2021

Edited by Bruno Cugny, Zoran Sodnik, and Nikos Karafolas
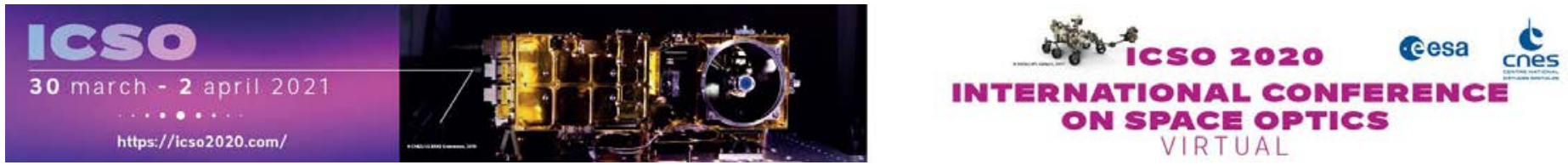

\section{Improvement of molecular adhesion for spatial applications at Thales SESO over the past 20 years}

\section{Cesa isoporeseatings denes}




\title{
Improvement of molecular adhesion for spatial applications at Thales SESO over the past 20 years \\ Natacha Cocheteau ${ }^{* a}$, Christian Du Jeu ${ }^{\mathrm{a}}$, Alizée Loarer ${ }^{\mathrm{a}}$, Patrick Robert ${ }^{\mathrm{a}}$, Mailys Thorigny ${ }^{\mathrm{a}}$ \\ ${ }^{a}$ Thales SESO, 530 rue Frédéric Joliot 13290 Aix-les-Milles, France
}

\begin{abstract}
Molecular adhesion is a well-known process used on terrestrial optics and other fields. This process consists in joining two surfaces without the use of any adhesive or additional material. Molecular adhesion is a high-precision production process, and assemblies obtained present a dimensional and thermal stability due to the absence of mechanical part or glue.

For over the past 20 years, Thales SESO developed its own process of molecular adhesion. For example among others, it has been applied on the manufacturing of Fabry-Perot interferometers. Such as fused silica Fabry-Perot for ALADIN program in the frame of AEOLUS mission (launched in August 2018) or fused silica/Zerodur ${ }^{\circledR}$ Fabry-Perot for ATLID program. Both interferometer have cavities under vacuum. On the ATLID Fabry-Perot the sealing is also made using molecular adhesion. Back then, the molecular adhesion admissible mechanical strength was $1 \mathrm{MPa}$.

Then, Thales SESO improved its molecular adhesion process - through lots of collaborations with CNES and other partners - in order to be able to integrate more complex designs and increase the mechanical resistance. Today the molecular adhesion admissible mechanical strength has been multiplied by a factor five.

Here after, you find an overview of the Thales SESO realizations starting from Fabry-Perot first results to the latest results on corner cubes performances as well as the future of molecular adhesion for space applications imagined by Thales SESO.
\end{abstract}

Keywords: molecular adherence, corner cube

\section{INTRODUCTION}

Also known as "direct bonding", "optical bonding", "optical contacting" or "wafer bonding", molecular adhesion is a well-known process and consists in joining two surfaces without any use of any adhesive or additional materials. Two solids with well-polished flat surfaces are brought into close proximity, they locally attract to each other by Van der Waals or hydrogen bonds and adhere or bond [1,2]. The main applications of this adherence process are on microelectronics devices and optics.

The basic phenomenon involved in molecular adhesion has been known and used for many centuries. Around 1230, the importance of surface cleaning during gold contacting has been noticed. The first discussion about solid adhesion seems to be related to an hypothetical experiment proposed by Galilee : if two surfaces perfectly flat and polished of glass or metal are brought into contact, they bond each other. Galilee affirms that this phenomenon is impossible in the case of rough surfaces. Moreover, the importance of polishing in adherence has been demonstrated by Desagulier in 1734. This phenomenon has been few used until mid-1980 where it appears in microelectronics and the SOI (Silicon-On-Insulator) manufacturing.

In the optical field, the first patent concerning molecular adhesion dates from 1920 and has been deposed by W.E. Williams on two interferometers : a Fabry Perot and a Michelson interferometer with blades made using molecular adhesion. More recently, Philips patented in 1962 this technology on a He-Ne laser with mirrors positioned at each extremity of the cavity and fixed using molecular adhesion.

The process is also applied in optical workshop to allow reaching accurate angles and flatness, by adhering the part to be manufactured onto a reference block 
Nowadays, this process is used in microelectronics on silicon-on-insulators devices, silicon based sensor and actuators, etc...[3]. In Optics, it is used in the manufacturing of complexes optical systems, to create assemblies (interferometers, slicers...). One example is the manufacturing slicers for the Multi Unit Spectroscopic Explorer for the Very Large Telescope [4].

Molecular adhesion is a high-precision production process, and obtained assemblies present a dimensional stability due to the absence of mechanical part or glue. In addition, since no adhesive materials are used in this process, the risks of contamination associated with degassing are avoided. Several materials can be adhered (fused silica, silicon, silicon carbide, zerodur ${ }^{\circledR}$, some metals, etc) and adherences can be used under cryogenic environment. Based on these benefits, this process is of particular interest for optical system manufacturing for spatial applications where involved constraints (thermal fatigue, accelerations, vibrations) are very different from those encountered on Earth.

This paper will first present how molecular adhesion works. Then, an overview of the Thales SESO realizations from terrestrial applications to space applications will be made explaining how - starting from simple realizations - the process has been improved to be spatially qualified and applied on lightweighted hollow corner cubes performances fully adhered.

\section{MODLECULAR DHESION: PRINCIPLE OF THE PROCESS}

Molecular adhesion consists in joining two surfaces without any use of any adhesive or additional materials. Direct bonding is possible as long as the two surfaces have similar geometries and requires clean surfaces free of contaminants $[5,6]$. Therefore, first steps of the adhesion process consist in physical and chemical preparation of surfaces:

- Several polishing of both surfaces to adhere were manually performed in order to obtain the exact surface roughness (here, roughness is always less than $1 \mathrm{~nm}$ RMS), flatness and deflection required. Indeed, a high level of roughness results in a weak contact zone and thus in the occurrence of defects during the bonding process. When the roughness is too great, bonding becomes impossible $[7,8]$.

- Then, surfaces undergo a chemical treatment in order to eliminate any contaminating particles [9]. With ambient humidity, free silicon surfaces are recovered by silanol $(\mathrm{Si}-\mathrm{OH})$ groups which are the precursor of the bonding [10 - 12]. With this chemical treatment, the more silanol groups possible on both surfaces is seek. On figure 2-1, one component of the roughness is represented, each line represents a bonding site, and obviously a lack of bonding sites causes incomplete bonding.
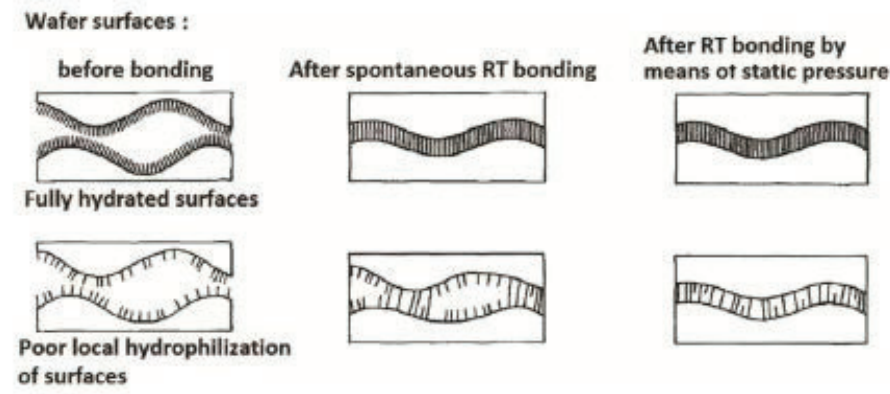

Figure 2-1: Behaviors of surfaces during room temperature bonding according to their hydroxylated rate [13]

Once physical preparation and surface treatment has been completed, surfaces are covers with clusters of water molecules due to ambient humidity. Then, surfaces are brought into contact and bonding occurs between water' clusters present on the two surfaces. By applying a slight local pressure, the surfaces are brought closer together, and hydrogen bonds developed between molecules of water present at the interface and the two surfaces. A bonding wave propagates from the contact point through the whole sample, resulting in the bonding of the surfaces. Molecules of water migrate from the bonding interface and/or diffuse at the interface, bringing the surfaces even closer together and initiating 
silanol-silanol covalent bonds [14-17]. Figure 2-2 shows the evolution of bonds during adherence starting from hydrogen bonds between clusters of water to covalent bonds for permanent sealing.

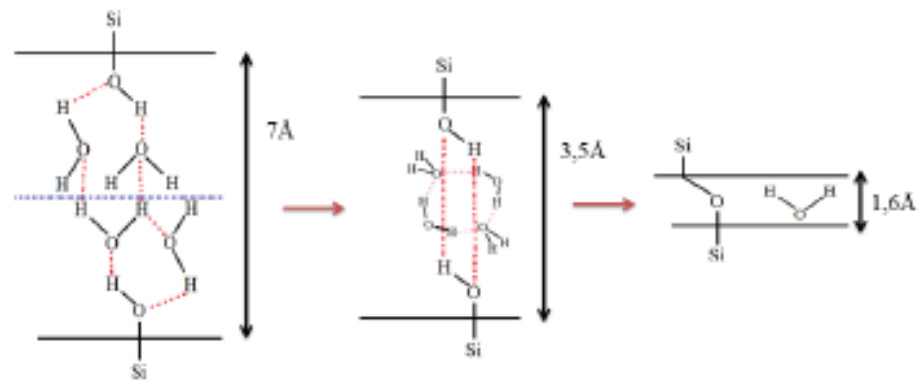

Figure 2-2: Evolution of bonds during molecular adherence process [17]

\section{MORE THAN 20 YEARS OF MOLECULAR ADHESION AT THALES SESO}

This is a commonly use process in optics. Indeed, it is used to maintain pieces on a support for precision polishing as shown in figure 3-1, for example:

- To polish a thin piece with high precision without inducing deformation

- To polish angles with precision
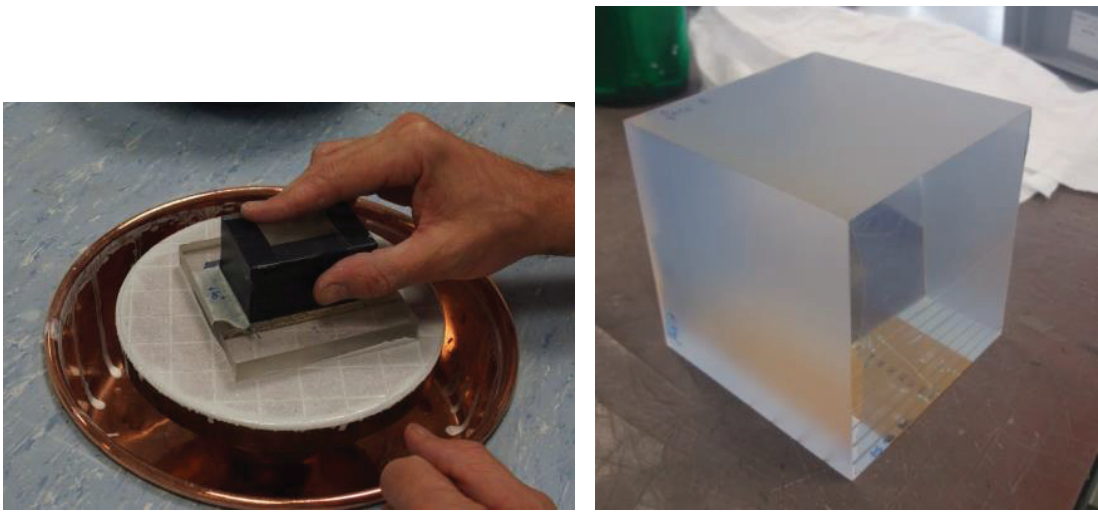

Figure 3-1: Example of adhered pieces for precision polishing

Obviously, for this kind of application, the adherence doesn't need to be too resistant as surfaces need to be separated after the manufacturing. However, by knowing precisely bonds involved in adherence the process can be optimized for weak adhesion.

With years, Thales SESO improved its process in order to adhered more complexes assemblies - used as tools - such as dihedral which are used to positions parts during bonding.

Then, Thales SESO manufactured interferometers for terrestrial applications using different materials - fused silica, Zerodur ${ }^{\circledR}$ - using molecular adherence for different applications. The double Fizeau interferometer presented in figure 32 is an example of assemblies manufactured. It has been manufactured using fused silica component. The angular tolerances obtained were $5 "$ and surfaces performances around $\lambda / 100 \mathrm{nmPTV}$. The figure 3-2 shows the optical components positioned on the base plate using molecular adhesion 


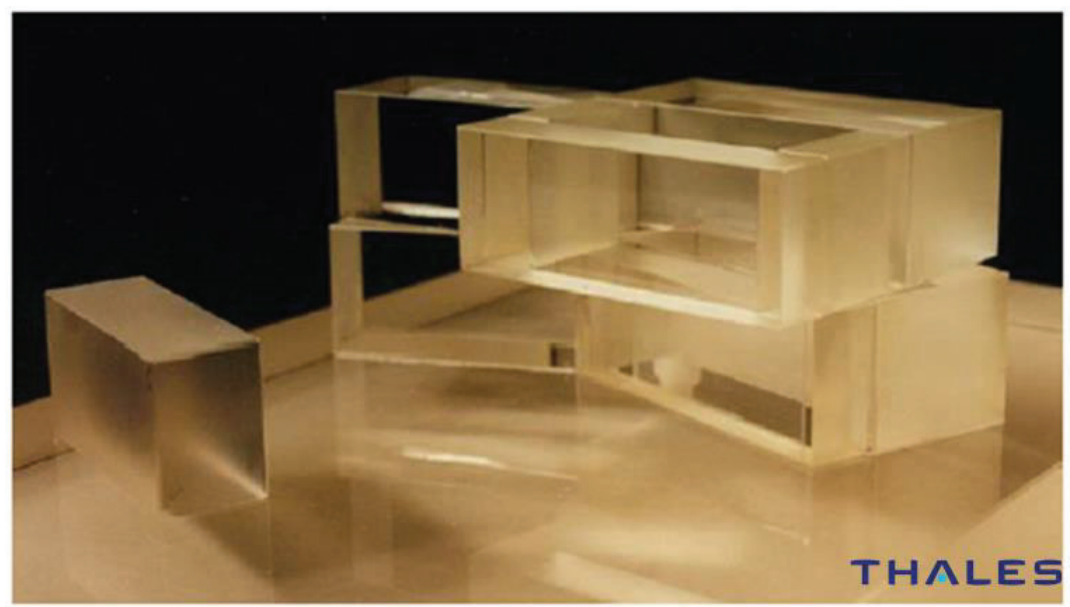

Figure 3-2:Double Fizeau interferometer

For terrestrial applications, the mechanical resistance of the adherence does not need to be resistant. Indeed, on ground application there is no requirement concerning shock or vibration. Nevertheless, for space application, the constraint involved (thermal fatigue, vibration, etc) are very different from those encountered on Earth. Thus, Thales SESO continued to improve its process in order to improve mechanical resistance.

In the early 2000, in the frame of the Aladin program - mission including a space lidar to measure windspeed- , Thales SESO developed :

- A Mie Fabry Perrot made out of fused silica plates and zerodur ${ }^{\circledR}$ spacer (MSPA)

- And a Rayleigh fused silica "Double Channel" Fabry-Perot assembly (RSP) qualified for Space Application

This double cavity Fabry Perot (Figure 3-3) includes :

- Double Fabry Perot with a difference of thickness of $80 \mathrm{~nm}$ between the two cavities. This Double Fabry-Perot has been realised on the same cavity.

- 4 prisms, 4 waveplates and one lens completed the optical setup.

- The Fabry-Perrot was also adhered on a base plate and its fixture. The complete assembly is shown on figure 33.

- The molecular adherence was reinforced using an external glue joint

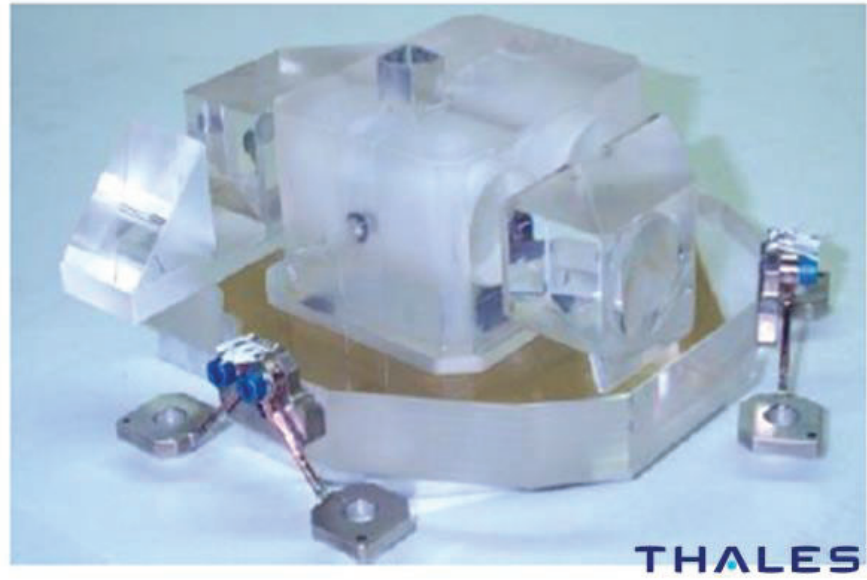

Figure 3-3: Double Fabry Perot for the Aladin program [18] 
The main specification, which were achieved are :

- Fabry Perot flatness of 2 areas of $26 \mathrm{~mm}$ diameter: $9 \mathrm{~nm}$ and $18 \mathrm{~nm}$

- Coating $\mathrm{R}=65 \pm 1 \%$; absorption $<0.3 \%$

- Finesse 65 at $355 \mathrm{~nm}$

- Assembly is able to pass:

- Static accelerations $>30 \mathrm{G}$ on all axes

- Thermal environment $-20^{\circ} \mathrm{C}$ to $50^{\circ} \mathrm{C}$.

Mission was launched in 2018 and the results according to ESA are very good ("ESA's Aeolus satellite has been returning profiles of Earth's winds since 3 September 2018, just after it was launched - and after months of careful testing these measurements are considered so good that the European Centre for Medium-Range Weather Forecasts is now using them in their forecasts").

Then in the frame of the LISA program, Thales SESO realized a cavity made with three mirror The stabilisation is based on the Pound Drever Hall or Tilt locking. The main part of this cavity is a cylinder of $138 \mathrm{~mm}$ length with a $95 \mathrm{~mm}$ diameter. Figure 3-4 shows the two flat surfaces of this cylinder 6 pieces, 7 and 2 are optically contacted. Piece 2 is a prism on which the mirrors 1 are optically contacted. A socket 5 is also contacted and on this support socket are cemented the sensors. With this cavity the goal is to obtain a frequency stability better than $10 \mathrm{~Hz} / \mathrm{sqrt}(\mathrm{Hz})$. The Finesse should be comprised between 10000 and 100000 .
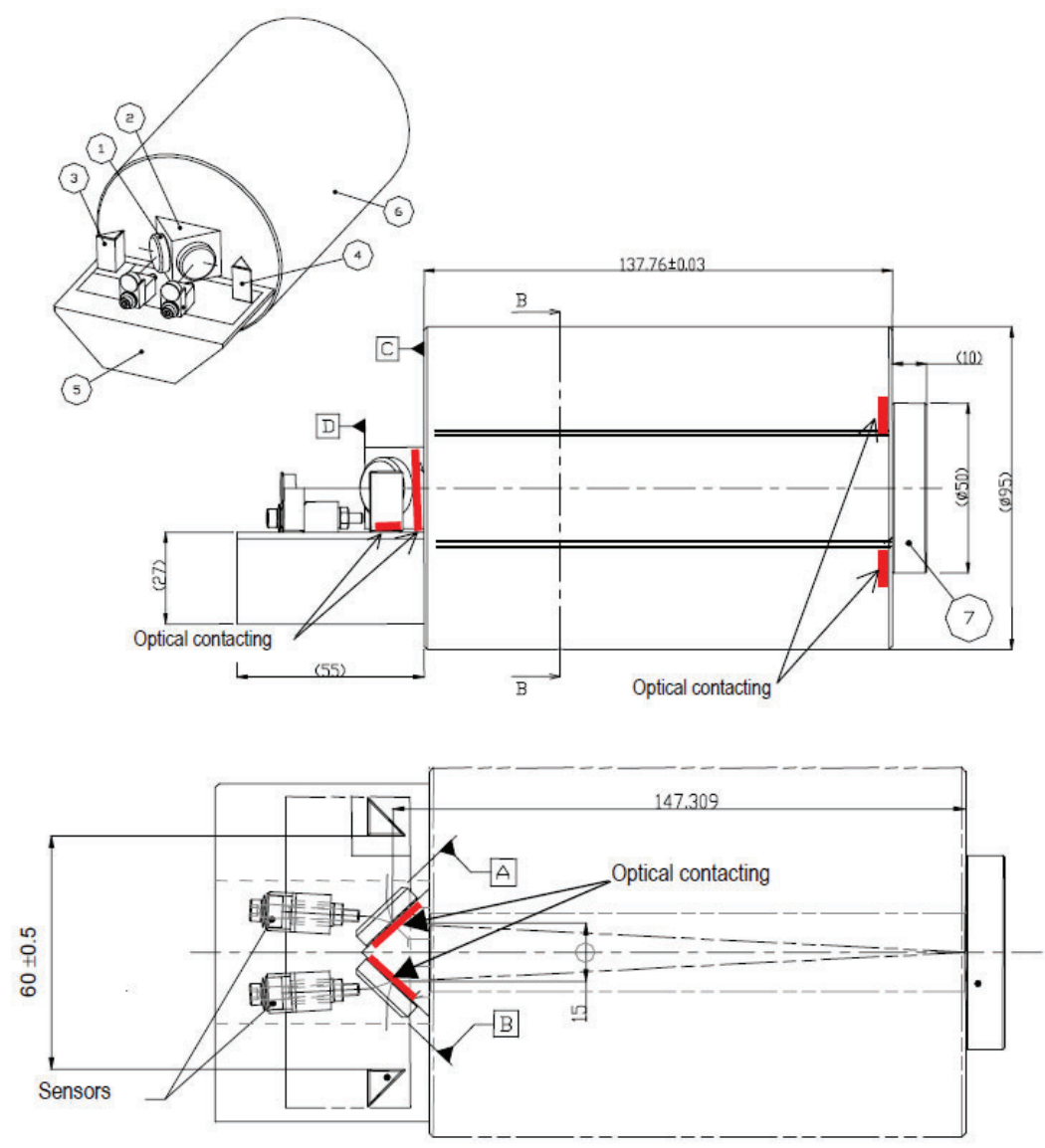

Figure 3-4: Lisa program cavity [18] 
Finally, in the frame of the satellite EarthCARE mission, Thales SESO developed the ATLID Fabry-Perot which consists in an assembly made with Zerodur ${ }^{\circledR}$ and fused silica with a cavity under vacuum (see figure 3-5). The sealing of the vacuum cavity was also made using molecular adhesion. For this application, external glue joint was forbidden.

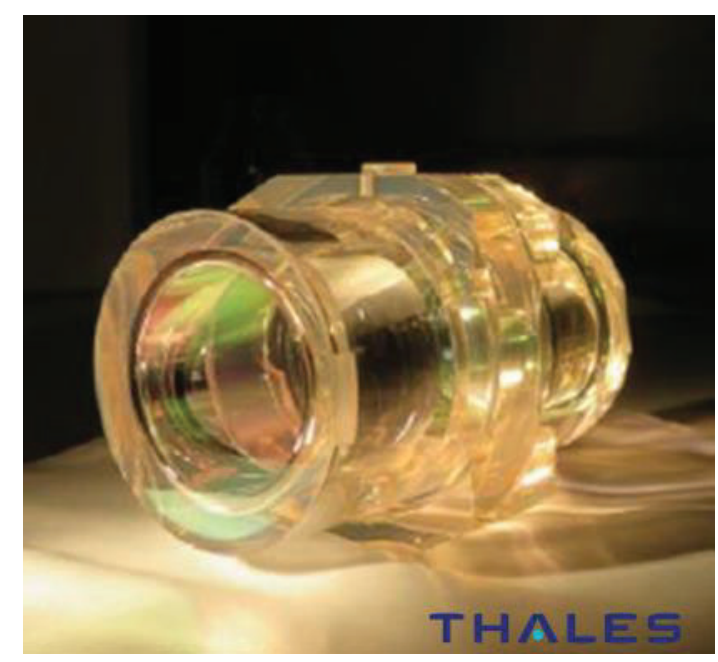

Figure 3-5: ATLID Fabry-Perot

With all this assemblies, Thales SESO qualified its molecular adherence process for space applications. However, shape of adhered surface was quite simple and mechanical resistance of assemblies obtained are around 1Mpa. Thus, the next step of its development was to adhere more complex geometries.

Through lot of collaborations with CNES and other partners, Thales SESO works on the development of cube corner assemblies using molecular adherence. This works lead to the spatial qualification of an hollow corner cube.

In the frame of the Meteorological Third Generation satellite (MTG) Thales SESO develop a large hollow lightweighted corner cube presented in Figure 3-6.

Indeed, the MTG-IRS interferometer is composed by two cube corner with very stringent specifications - twice stringent compared to the actual knowledge - with high stability, mechanical resistance in accordance with mechanical and thermal environment of fixed geostationary orbit with following optical performances:

- Each angle of the cube corner need to be at $90^{\circ} \pm 1$,

- Wave Front Error (WFE) $<250$ nm RMS

- $\quad$ Mass $<250 \mathrm{~g}$

After lots of development and characterization [19], Thales SESO succeed to increase the mechanical resistance of the molecular adhesion by a factor five. The cube corner is composed by three fused silica mirrors adhered one to the other. The assembly is bonded onto a mechanical bracket.

The results is compliant with the optical and mass specifications:

- WFE achieved is less than $250 \mathrm{~nm}$ RMS on $\Phi 78 \mathrm{~mm}$

- Total mass is $245 \mathrm{~g}$

Then, this cube corner has been fully qualified, which means it has been submitted to:

- Dynamic test:

○ 60 gRMS in sine test

- Random solicitations 
○ Shock solicitations $(123 \mathrm{~g})$

- Thermal test
$\circ$ Between $-12^{\circ}$ and $+40^{\circ}$

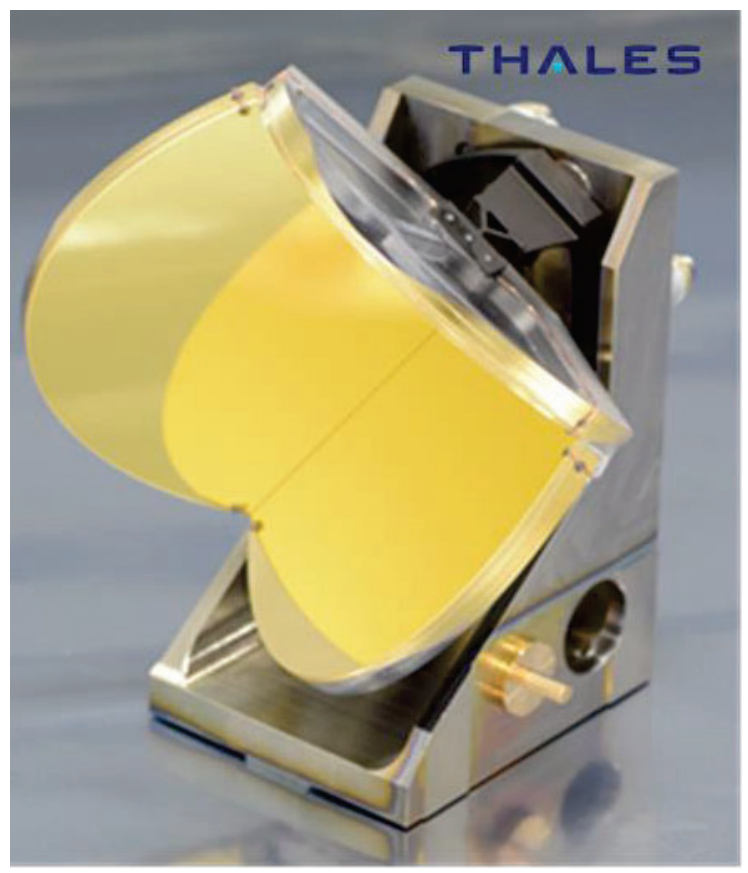

Figure 3-6: The two corner cubes developed in the frame of MTG-IRS

Moreover, the cube corner demonstrate during test a high WFE stability under vacuum as presented in the following figure.

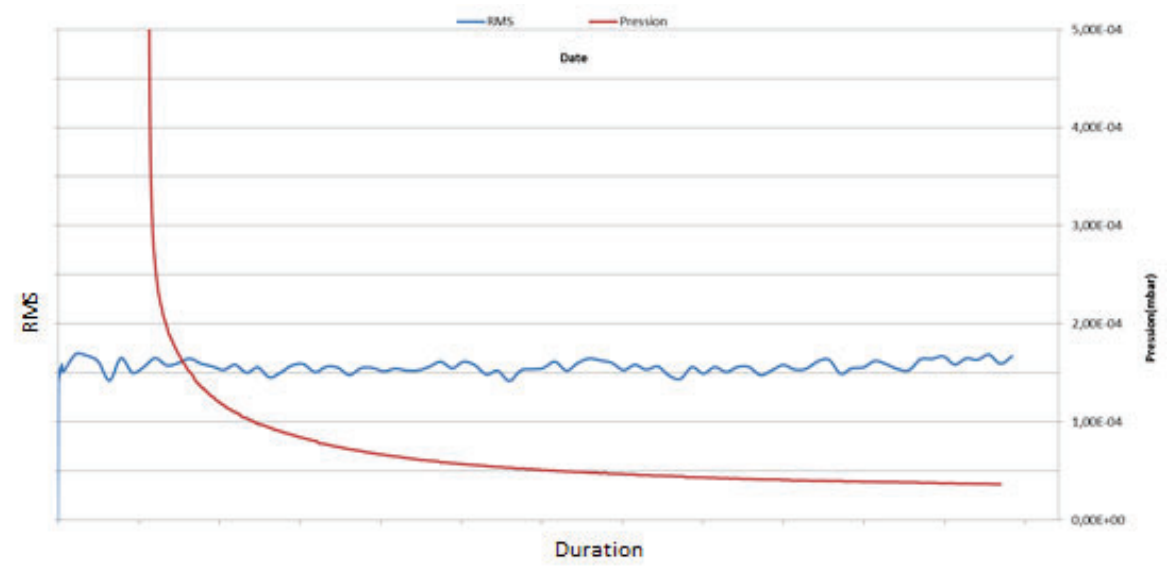

Figure 3-7: Evolution of WFE under vacuum (red curve : pressure and blue curve : WFE) 


\section{WHAT'S NEXT?}

The main challenge now is to industrialize the production in order to manufactured series of corner cubes.

Industrialization of corner cubes need other improvements of molecular adherence, in order to have a repeatable process for series manufacturing. Today, each mirror of the corner cubes are perfectly prepared before adherence with very low WFE and angles at $90 \pm 0.5$ ". The major difficulty comes from the adherence of the third mirror. Thus, the industrialization challenge will be focused on solving this issue.

\section{REFERENCES}

[1] Tong Q.Y., Gösele U., "Semiconductor wafer bonding: recent developments", Material Chemistry and Physics, 37, 101-127(1994)

[2] Kendall K., [Molecular adhesion and its applications]. Kluwer Academic Publishers (2001)

[3] Berthold A., Vellekoop M., "IC-compatible silicon wafer-to-wafer bonding", Sensors and Actuators, A: Physical, 60, 208-2011 (1997)

[4] Laurent F., "MUSE Image Slicer: Test results on largest slicer ever manufactured", Proc. SPIE 7018, 71, 7018-7030 (2008)

[5] Yu H.H., Suo Z., “ A model of wafer bonding by elastic accommodation”, Journal of Mechanical Physics and Solids, 46(5), 829-844 (1998)

[6] Rayssac O., Olivier M., Stoemenos I., Cartier A.M., Aspar B., "Silicon-on-Insulator technology and devices X", The electrochemical society proceedings series, Pennington NJ, 39, PV01-03 (2001)

[7] Tang Z., "Modelling the formation of spontaneous wafer direct bonding under low temperature", Microelectronic Engineering, 85, 1754-1757 (2001)

[8] Liao G., Lin X., "Surface roughness modeling for silicon direct bonding", IEEE transaction on components, packaging and manufacturing technology, 1(8), 1171 - 1177 (2011)

[9] Lai S., Lin H., Yu C., "Effect of surface treatment on wafer direct bonding process", Material Chemistry and Physics, 83, 265-272 (2004)

[10] Klier K., Zettlemoyer A.C. , "Water at interface: Molecular structure and dynamics", Journal of Colloid and Interface Science, 58(2), 216-229 (1977)

[11] Iler R. K., [The chemistry of Silica], Wiley (1979)

[12] Cocheteau N., Maurel-Pantel A., Lebon F., Rosu I., Ait Zaid S., Savin Delarclause I., "Influence of process parameters on the mechanical resistance of direct bonded silica glasses", Journal of Adhesion Science and Technology, 28(10), 915-934 (2014)

[13] Kissinger G., Kissinger W., "Void-free silicon-wafer-bond strengthening in the $200-400^{\circ} \mathrm{C}$ range", Sensors and Actuators, A: Physical, 36, 149-156 (1993)

[14] Stengl R., Tan T., Gösele U., "A model for the silicon wafer bonding process", Japanese Journal of Applied Physics, 28, 10:1735-1741 (1977)

[15] Ventosa C., Rieutord, F., Libralesso, L., Morales, C., Fournel, F., Moriceau, H., "Hydrophilic low-temperature direct wafer bonding", Journal of Applied Physics, 104, 123524-123526 (2008)

[16] Ventosa C., Morales C., Libralesso L., Fournel F., Papon A.M., Lafond D., Moriceau H., Penot J. D., Rieutord F., "Mechanism of thermal silicon oxide direct wafer bonding", Electrochemical and Solid States Letters, 12(10), 375379 (2009)

[17] Cocheteau N., “Caractérisation et modélisation d'une adherence moléculaire renforcée”, Ph.D thesis, Aix-Marseille University, 2014

[18] Fermé J.J., "Optical contacting”, Optical system Design (2003)

[19] Cocheteau N., Du Jeu C., Voisin M., Maurel-Pantel A., "Shock and vibration tests of space light-weighted corner cubes manufactured with adherence process", Proc. SPIE 11180, 111808B (2018)

[20] Fermé J.J., Gasc K., "High stability hollow cube corner”, Proc. SPIE 10566. 105661V (2017) 